Combinatorics, Probability and Computing (2018. This is an Open Access article, distributed under the terms of the Creative Commons Attribution licence (http://creativecommons.org/licenses/by/4.0/), which permits unrestricted re-use, distribution, and reproduction in any medium, provided the original work is properly cited.) 27, 725-740. C Cambridge University Press 2018

\title{
Packing Graphs of Bounded Codegree
}

\author{
WOUTER CAMES VAN BATENBURG ${ }^{\dagger}$ and ROSS J. KANG ${ }^{\ddagger}$ \\ Department of Mathematics, Radboud University Nijmegen, PO Box 9010, 6500 GL Nijmegen, Netherlands \\ (e-mail: w.camesvanbatenburg@math.ru.nl, ross.kang@gmail.com)
}

Received 18 May 2016; revised 28 November 2017; first published online 22 March 2018

\begin{abstract}
Two graphs $G_{1}$ and $G_{2}$ on $n$ vertices are said to pack if there exist injective mappings of their vertex sets into $[n]$ such that the images of their edge sets are disjoint. A longstanding conjecture due to Bollobás and Eldridge and, independently, Catlin, asserts that if $\left(\Delta\left(G_{1}\right)+1\right)\left(\Delta\left(G_{2}\right)+1\right) \leqslant n+1$, then $G_{1}$ and $G_{2}$ pack. We consider the validity of this assertion under the additional assumption that $G_{1}$ or $G_{2}$ has bounded codegree. In particular, we prove for all $t \geqslant 2$ that if $G_{1}$ contains no copy of the complete bipartite graph $K_{2, t}$ and $\Delta\left(G_{1}\right)>17 t \cdot \Delta\left(G_{2}\right)$, then $\left(\Delta\left(G_{1}\right)+1\right)\left(\Delta\left(G_{2}\right)+1\right) \leqslant n+1$ implies that $G_{1}$ and $G_{2}$ pack. We also provide a mild improvement if moreover $G_{2}$ contains no copy of the complete tripartite graph $K_{1,1, s}, s \geqslant 1$.
\end{abstract}

2010 Mathematics subject classification: Primary 05C70

Secondary $05 \mathrm{C} 35$

\section{Introduction}

Let $G_{1}$ and $G_{2}$ be graphs on $n$ vertices. (All graphs are assumed to have neither loops nor multiple edges.) We say that $G_{1}$ and $G_{2}$ pack if there exist injective mappings of their vertex sets into $[n]=\{1, \ldots, n\}$ so that their edge sets have disjoint images. Equivalently, $G_{1}$ and $G_{2}$ pack if $G_{1}$ is a subgraph of the complement of $G_{2}$. The maximum codegree $\Delta^{\wedge}(G)$ of a graph $G$ is the maximum over all vertex pairs of their common degree, i.e. $\Delta^{\wedge}(G)<t$ if and only if $G$ contains no copy of the complete bipartite graph $K_{2, t}$. The maximum adjacent codegree $\Delta^{\Delta}(G)$ of $G$ is the maximum over all pairs of adjacent vertices of their common degree, i.e. $\Delta^{\Delta}(G)<s$ if and only if $G$ contains no copy of the complete tripartite graph $K_{1,1, s^{*}}$. Clearly, $\Delta^{\Delta}(G) \leqslant \Delta^{\wedge}(G)$ always. We let $\Delta_{1}$ and $\Delta_{2}$ denote the maximum degrees of $G_{1}$ and $G_{2}$, respectively, and $\Delta_{1}^{\wedge}$ and $\Delta_{2}^{\Delta}$ the corresponding maximum (adjacent) codegrees. We provide sufficient conditions for $G_{1}$ and $G_{2}$ to pack in terms of $\Delta_{1}, \Delta_{2}, \Delta_{1}^{\wedge}, \Delta_{2}^{\Delta}$.

\footnotetext{
$\dagger$ Supported by NWO grant 613.001.217.

$\ddagger$ Supported by a NWO Vidi grant (639.032.614).
} 
For integers $t \geqslant 2$ and $\Delta_{2} \geqslant 1$, we define

$$
\alpha^{*}\left(t, \Delta_{2}\right):=\frac{1}{2}\left(2+\gamma+\sqrt{4 \gamma+\gamma^{2}}\right), \text { where } \gamma=\frac{\Delta_{2}}{\Delta_{2}+1} \cdot \frac{t-1}{t} .
$$

Note $\alpha^{*}=\alpha^{*}\left(t, \Delta_{2}\right)$ is the larger solution to the equation $(\alpha-1)^{2}-\gamma \alpha=0$ and

$$
\frac{1}{8}(9+\sqrt{17}) \leqslant \alpha \leqslant \frac{1}{2}(3+\sqrt{5}) .
$$

Theorem 1.1. Let $G_{1}$ and $G_{2}$ be graphs on $n$ vertices with respective maximum degrees $\Delta_{1}$ and $\Delta_{2}$. Let $\Delta_{1}^{\wedge}$ be the maximum codegree of $G_{1}$. Let $t \geqslant 2$ be an integer and let $\alpha>\alpha^{*}=\alpha^{*}\left(t, \Delta_{2}\right)$ and $0<\varepsilon<1 / 2$ be reals. Then $G_{1}$ and $G_{2}$ pack if $\Delta_{1}^{\wedge}<t$ and $n$ is larger than each of the following quantities:

$$
\begin{gathered}
\left(t+\frac{\alpha(\alpha-1)}{(\alpha-1)^{2}-\alpha}\right) \cdot \Delta_{2}+\Delta_{1} \Delta_{2}, \\
(2 \alpha t+2) \cdot \Delta_{2}+((2 \alpha+1) t-1) \cdot \Delta_{2}^{2}+(1-\varepsilon) \cdot \Delta_{1} \Delta_{2}, \\
1+\left(2+\frac{\varepsilon}{1-2 \varepsilon}\right) \cdot \Delta_{2}+\Delta_{1} \Delta_{2}, \quad \text { and } \\
\left(t+\frac{3-\varepsilon}{2}\right) \cdot \Delta_{2}+\frac{3-\varepsilon}{2}(t-1) \cdot \Delta_{2}^{2}+\frac{1+\varepsilon}{2} \cdot \Delta_{1} \Delta_{2} .
\end{gathered}
$$

Theorem 1.2. Let $G_{1}$ and $G_{2}$ be graphs on $n$ vertices with respective maximum degrees $\Delta_{1}$ and $\Delta_{2}$. Let $\Delta_{1}^{\wedge}$ be the maximum codegree of $G_{1}$ and $\Delta_{2}^{\Delta}$ the maximum adjacent codegree of $G_{2}$. Let $s \geqslant 1$ and $t \geqslant 2$ be integers and let $\alpha>\alpha^{*}=\alpha^{*}\left(t, \Delta_{2}\right)$ be real. Then $G_{1}$ and $G_{2}$ pack if $\Delta_{1}^{\wedge}<t$, $\Delta_{2}^{\Delta}<s$, and $n$ is larger than both of the following quantities:

$$
\begin{array}{r}
\left(t+\frac{\alpha(\alpha-1)}{(\alpha-1)^{2}-\alpha}\right) \cdot \Delta_{2}+\Delta_{1} \Delta_{2} \quad \text { and } \\
(2+2 \alpha t) \cdot \Delta_{2}+(s-1) \cdot \Delta_{1}+((2 \alpha+1) t-1) \cdot \Delta_{2}^{2} .
\end{array}
$$

For better context, we compare Theorems 1.1 and 1.2 to a line of work on graph packing that was initiated in the 1970s $[2,6,7,17]$. The following is a central problem in the area.

Conjecture 1.3 (Bollobás and Eldridge [2] and Catlin [7]). Let $G_{1}$ and $G_{2}$ be graphs on $n$ vertices with respective maximum degrees $\Delta_{1}$ and $\Delta_{2}$. Then $G_{1}$ and $G_{2}$ pack if $\left(\Delta_{1}+1\right)\left(\Delta_{2}+1\right) \leqslant$ $n+1$.

If true, the statement would be sharp and would significantly generalize a celebrated result of Hajnal and Szemerédi [12] on equitable colourings. Sauer and Spencer [17] showed that $2 \Delta_{1} \Delta_{2}<$ $n$ is a sufficient condition for $G_{1}$ and $G_{2}$ to pack, which is seen to be sharp when one of the graphs is a perfect matching. Thus far the Bollobás-Eldridge-Catlin (BEC) conjecture has been confirmed in the following special cases: $\Delta_{1}=2$ [1]; $\Delta_{1}=3$ and $n$ sufficiently large [10]; $G_{1}$ bipartite and $n$ sufficiently large [9]; and $G_{1} d$-degenerate, $\Delta_{1} \geqslant 40 d$ and $\Delta_{2} \geqslant 215$ [5]. We would 
also like to highlight the following three results that can be considered approximate forms of the BEC conjecture. (a) The condition $\left(\Delta_{1}+1\right)\left(\Delta_{2}+1\right) \leqslant 3 n / 5+1$ is sufficient for $G_{1}$ and $G_{2}$ to pack, provided that $\Delta_{1}, \Delta_{2} \geqslant 300$ [15]. (b) The BEC condition is sufficient for $G_{1}$ and $G_{2}$ to admit a 'near packing' in that the subgraph induced by the intersection of their images has maximum degree at most 1 [11]. (c) If $G_{2}$ is chosen as a binomial random graph of parameters $n$ and $p$ such that $n p$ in place of $\Delta_{2}$ satisfies the BEC condition, then $G_{1}$ and $G_{2}$ pack with probability tending to 1 as $n \rightarrow \infty$ [3].

Corollary 1.4. Let $G_{1}, G_{2}, \Delta_{1}, \Delta_{2}$ and $\Delta_{1}^{\wedge}$ be as before. Let $t \geqslant 2$ be an integer. Then $G_{1}$ and $G_{2}$ pack if $\Delta_{1} \Delta_{2}+\Delta_{1} \leqslant n+1$ and $\Delta_{1}^{\wedge}<t$ and $\Delta_{1}>17 t \cdot \Delta_{2}$.

Proof. Choose $\varepsilon=(2 t-2) /(4 t-3)$ and $\alpha=3$ in Theorem 1.1. Using that

$$
\Delta_{1}>17 t \Delta_{2}>\frac{(4 t-3)(7 t-1)}{2 t-2} \cdot \Delta_{2}
$$

it follows that $\max ((1.1),(1.2),(1.3),(1.4)) \leqslant\left(\Delta_{1}+1\right)\left(\Delta_{2}+1\right)-1 \leqslant n$. So $G_{1}$ and $G_{2}$ pack. $\square$

We have the following results concerning the BEC conjecture.

Corollary 1.5. Given an integer $t \geqslant 2$, the BEC conjecture holds under the additional condition that the maximum codegree $\Delta_{1}^{\wedge}$ of $G_{1}$ is less than $t$ and $\Delta_{1}>17 t \cdot \Delta_{2}$.

We were unable to avoid the linear dependence on $\Delta_{2}$ in the lower bound condition on $\Delta_{1}$. Although we have not seriously attempted to optimize the factor $17 t$ above, Theorem $1.2 \mathrm{im}$ proves on this factor under the additional assumption that $\Delta_{2}^{\Delta}$ is bounded, as exemplified by the following corollary.

Corollary 1.6. Given an integer $t \geqslant 2$, the BEC conjecture holds under the additional condition that the maximum codegree $\Delta_{1}^{\wedge}$ of $G_{1}$ is less than $t, G_{2}$ is triangle-free, and $\Delta_{1}>(4+\sqrt{5}) t \cdot \Delta_{2}$.

Proof. Choose

$$
\alpha=\frac{1}{4 t}\left(6 t+1+\sqrt{20 t^{2}+4 t+1}\right)
$$

and $s=1$ in Theorem 1.2. Using that

$$
t+\frac{\alpha(\alpha-1)}{(\alpha-1)^{2}-\alpha}-1=(2 \alpha+1) t-1
$$

and that $\Delta_{1}>(4+\sqrt{5}) t \cdot \Delta_{2}>((2 \alpha+1) t-1) \cdot \Delta_{2}$, it follows that $\max ((1.5),(1.6)) \leqslant\left(\Delta_{1}+\right.$ 1) $\left(\Delta_{2}+1\right)-1 \leqslant n$. So $G_{1}$ and $G_{2}$ pack.

\section{Structure of the paper}

In the next section, we provide some notation and preliminary observations. In Section 3, we discuss the common features of a hypothetical critical counterexample to one of our theorems. In Section 4, we prove Theorems 1.1 and 1.2. We conclude the paper with some remarks about the results, proofs and further possibilities. 


\section{Notation and preliminaries}

Here we introduce some terminology which we use throughout. We often call $G_{1}$ the blue graph and $G_{2}$ the red graph. We treat the injective vertex mappings as labellings of the vertices from 1 to $n$. However, rather than saying, 'the vertex in $G_{1}$ (or $G_{2}$ ) corresponding to the label $i$ ', we often only say, 'vertex $i$ ', since this should never cause any confusion. Our proofs rely on accurately specifying the neighbourhood structure as viewed from a particular vertex. Let $i \in[n]$. The blue neighbourhood $N_{1}(i)$ of $i$ is the set $\left\{j \mid i j \in E\left(G_{1}\right)\right\}$ and the blue degree $\operatorname{deg}_{1}(i)$ of $i$ is $\left|N_{1}(i)\right|$. The red neighbourhood $N_{2}(i)$ and red degree $N_{2}(i)$ are defined analogously. For $j \in[n]$, a red-blue link (or 2-1 link) from $i$ to $j$ is a vertex $i^{\prime}$ such that $i i^{\prime} \in E\left(G_{2}\right)$ and $i^{\prime} j \in E\left(G_{1}\right)$. The red-blue neighbourhood $N_{1}\left(N_{2}(i)\right)$ of $i$ is the set $\{j \mid \exists$ red-blue link from $i$ to $j\}$. A blue-red link (or 1-2 link) and the blue-red neighbourhood $N_{2}\left(N_{1}(i)\right)$ are defined analogously.

In search of a certificate that $G_{1}$ and $G_{2}$ pack, without loss of generality, we keep the vertex labelling of the blue graph $G_{1}$ fixed, and permute only the labels in the red graph $G_{2}$. This can be thought of as 'moving' the red graph above a fixed ground set $[n]$. In particular, we seek to avoid the situation that there are $i, j \in[n]$ for which $i j$ is an edge in both $G_{1}$ and $G_{2}-$ in this situation, we call $i j$ a purple edge induced by the labellings of $G_{1}$ and $G_{2}$. So $G_{1}$ and $G_{2}$ pack if and only if they admit a pair of vertex labellings that induces no purple edge. In our search, we make small cyclic sub-permutations of the labels (of $G_{2}$ ), which are referred to as follows. For $i_{0}, \ldots, i_{\ell-1} \in[n]$, a $\left(i_{0}, \ldots, i_{\ell-1}\right)$-swap is a relabelling of $G_{2}$ so that for each $k \in\{0, \ldots, \ell-1\}$ the vertex labelled $i_{k}$ is re-assigned the label $i_{k+1 \bmod \ell}$. In fact, we shall only require swaps having $\ell \in\{1,2\}$. The following observation describes when a swap could be helpful in the search for a packing certificate. This is identical to Lemma 1 in [15].

Lemma 2.1. Let $u_{0}, \ldots, u_{\ell-1} \in[n]$. For every $k, k^{\prime} \in\{0, \ldots, \ell-1\}$, suppose that there is no red-blue link from $u_{k}$ to $u_{k+1 \bmod \ell}$ and that if $u_{k} u_{k^{\prime}} \in E\left(G_{2}\right)$, then $u_{(k+1 \bmod \ell)} u_{\left(k^{\prime}+1 \bmod \ell\right)} \notin E\left(G_{1}\right)$. Then there is no purple edge incident to any of $u_{0}, \ldots, u_{\ell-1}$ after $a\left(u_{0}, \ldots, u_{\ell-1}\right)$-swap.

We will use a classic extremal set theoretic result to upper-bound the size of certain vertex subsets.

Lemma 2.2 (Corrádi [8]). Let $A_{1}, \ldots, A_{N}$ be k-element sets and $X$ be their union. If $\left|A_{i} \cap A_{j}\right| \leqslant$ $t-1$ for all $i \neq j$, then $|X| \geqslant k^{2} N /(k+(N-1)(t-1))$.

In particular, this implies the following.

Corollary 2.3. Let $A_{1}, \ldots, A_{N}$ be size $\geqslant k$ subsets of a set $X$. If $k^{2}>(t-1) \cdot|X|$ and $\left|A_{i} \cap A_{j}\right| \leqslant$ $t-1$ for all $i \neq j$, then

$$
N \leqslant|X| \cdot \frac{k-(t-1)}{k^{2}-(t-1) \cdot|X|} .
$$

Proof. Consider arbitrary subsets $A_{1}^{*} \subset A_{1}, \ldots, A_{N}^{*} \subset A_{N}$ of size $k$. An application of Corrádi's lemma to $A_{1}^{*}, \ldots, A_{N}^{*}$ yields that $|X| \geqslant k^{2} \cdot N /(k+(N-1)(t-1))$, which is easily seen to be equivalent to $\left(k^{2}-(t-1) \cdot|X|\right) \cdot N \leqslant(k-t+1) \cdot|X|$. The corollary follows after dividing both 
sides of the inequality by $k^{2}-(t-1) \cdot|X|$. Note that this division does not cause a sign change because of the assumption that $k^{2}>(t-1) \cdot|X|$.

\section{Hypothetical critical counterexamples}

The overall proof structure we use for both theorems is the same, and in this section we describe common features and some further notation. Suppose the theorem (one of Theorem 1.1 or 1.2) is false. Then there must exist a counterexample, that is, a pair $\left(G_{1}, G_{2}\right)$ of non-packable graphs on $n$ vertices that satisfy the conditions of the theorem.

Moreover, we may assume that $\left(G_{1}, G_{2}\right)$ is a critical pair in the sense that $G_{2}$ is edge-minimal among all counterexamples. In other words, $G_{1}$ and $G_{2}-e$ pack for any $e \in E\left(G_{2}\right)$. There is no loss of generality, since the removal of an edge from $G_{2}$ increases neither $\Delta_{2}$ nor $\Delta_{2}^{\Delta}$ and obviously affects none of $\Delta_{1}, \Delta_{1}^{\wedge}$ and $n$, thus maintaining the required conditions.

Now choose any edge $e=u v \in E\left(G_{2}\right)$. Criticality implies that there is a pair of labellings of $G_{1}$ and $G_{2}$ such that $e$ is the unique purple edge, for otherwise $G_{1}$ and $G_{2}-e$ do not pack. Let us fix such a pair of labellings so that we can further describe the neighbourhood structure as viewed from $u$ (or $v$ ). Estimation of the sizes of subsets in this neighbourhood structure is our main method for deriving upper bounds on $n$ that in turn yield the desired contradiction from which the theorem follows.

We need the definition of the following vertex subsets (which are analogously defined for $v$ also):

$$
\begin{aligned}
A(u) & :=N_{2}\left(N_{1}(u)\right) \backslash\left(N_{1}(u) \cup N_{2}(u) \cup N_{1}\left(N_{2}(u)\right)\right), \\
B(u) & :=N_{1}\left(N_{2}(u)\right) \backslash\left(N_{1}(u) \cup N_{2}(u) \cup N_{2}\left(N_{1}(u)\right)\right), \\
A^{*}(u) & :=N_{2}\left(N_{1}(u)\right) \backslash\left(N_{2}(u) \cup N_{1}\left(N_{2}(u)\right)\right), \quad \text { and } \\
N_{1}^{*}(u) & :=N_{1}(u) \cap\left(N_{1}\left(N_{2}(u)\right) \backslash\left(N_{2}(u) \cup N_{2}\left(N_{1}(u)\right)\right)\right) .
\end{aligned}
$$

One justification for specifying the above subsets is that the following two claims (which are essentially Claims 1 and 2 in [15]) hold.

Claim 3.1. For all $w \in[n] \backslash\{v\}$, there is a red-blue link or a blue-red link from $u$ to $w$.

Proof. If not, then by Lemma 2.1, a $(u, w)$-swap yields a new labelling such that $u v$ is no longer purple and no new purple edges are created. Thus $G_{1}$ and $G_{2}$ pack, a contradiction. See Figure 1.

Claim 3.2. For all $a \in A^{*}(u)$ and $b \in B(u)$, there is a red-blue link from $a$ to $b$.

Proof. Since $B(u) \cap N_{1}(u)=B(u) \cap N_{2}(u)=\emptyset$ and $A^{*}(u) \cap N_{2}(u)=\emptyset$, we have that $b u \notin$ $E\left(G_{1}\right) \cup E\left(G_{2}\right)$ and $u a \notin E\left(G_{2}\right)$. Furthermore, since $A^{*}(u) \cap N_{1}\left(N_{2}(u)\right)=B(u) \cap N_{2}\left(N_{1}(u)\right)=\emptyset$, there is no red-blue link from $u$ to $a$ or from $b$ to $u$. Now suppose that there is also no red-blue link from $a$ to $b$. Then it follows from Lemma 2.1 that after a $(u, a, b)$-swap there is no purple edge incident to any of $u, a, b$, which implies that there is no purple edge at all. So we have obtained a packing of $G_{1}$ and $G_{2}$, a contradiction. 


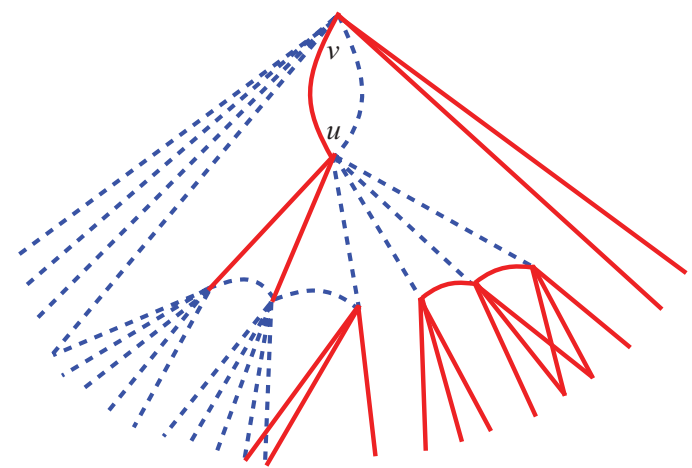

Figure 1. All vertices (except possibly $v$ ) are reachable by a link from $u$ (Claim 3.1).

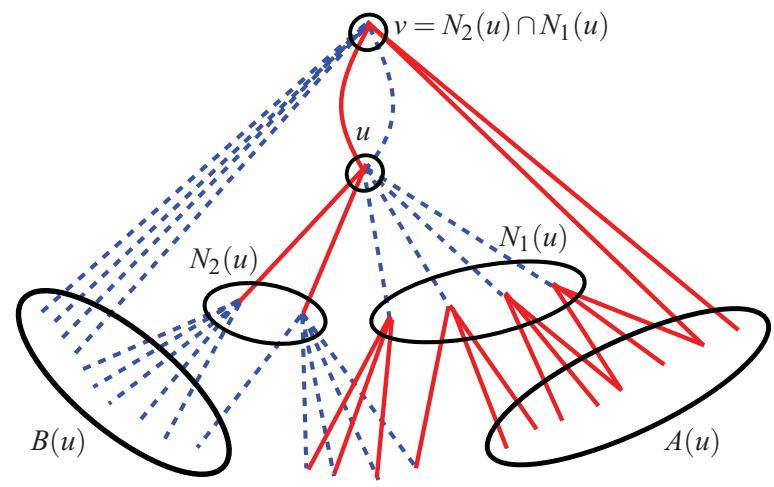

Figure 2. The neighbourhood structure of a hypothetical critical counterexample, as seen from $u$.

\section{See Figure 2}

In the next claim, we list three upper bounds on the total number $n$ of vertices in terms of the sizes of the vertex subsets defined above. In the proofs of Theorems 1.1 and 1.2, we consider several cases for which we prove at least one of these upper bounds to be small enough for a contradiction with the assumed lower bounds on $n$.

Claim 3.3. The total number $n$ of vertices is at most each of the following quantities:

(i) $\left|N_{2}(u)\right|+\left|A^{*}(u)\right|+\left|N_{1}\left(N_{2}(u)\right)\right|$,

(ii) $\left|N_{1}^{*}(u)\right|+\left|N_{2}(u)\right|+|B(u)|+\left|N_{2}\left(N_{1}(u)\right)\right|$,

(iii) $\left|A^{*}(v)\right|+\left|A^{*}(u)\right|+\left|\left(N_{2}(u) \cup N_{1}\left(N_{2}(u)\right)\right) \cap\left(N_{2}(v) \cup N_{1}\left(N_{2}(v)\right)\right)\right|$.

Proof. In all cases, $[n]$ equals the union of the neighbourhood sets that occur in the upper bound.

(i) The union of $N_{2}(u), A^{*}(u)$ and $N_{1}\left(N_{2}(u)\right)$ covers $\{v\} \cup N_{2}\left(N_{1}(u)\right) \cup N_{1}\left(N_{2}(u)\right)$, which by Claim 3.1 equals $[n]$.

(ii) The union of $N_{1}^{*}(u), N_{2}(u), B(u)$ and $N_{2}\left(N_{1}(u)\right)$ covers $\{v\} \cup N_{2}\left(N_{1}(u)\right) \cup N_{1}\left(N_{2}(u)\right)$, which equals $[n]$. 
(iii) By the proof of (i), $[n]$ is the union of $A^{*}(u)$ and $N_{2}(u) \cup N_{1}\left(N_{2}(u)\right)$ as well as the union of $A^{*}(v)$ and $N_{2}(v) \cup N_{1}\left(N_{2}(v)\right)$. It follows that $[n]$ also is the union of $A^{*}(u), A^{*}(v)$ and $\left(N_{2}(u) \cup N_{1}\left(N_{2}(u)\right)\right) \cap\left(N_{2}(v) \cup N_{1}\left(N_{2}(v)\right)\right)$.

The reason for working with $N_{1}^{*}(u)$ and $A^{*}(u)$ rather than the simpler sets $N_{1}(u)$ and $A(u)$ is the following. Under the requirement that the codegree $\Delta_{1}^{\wedge}$ of $G_{1}$ is less than $t$, we can upper-bound $\left|N_{1}^{*}(u)\right|$ entirely in terms of $\Delta_{2}$. This is sharper than the trivial bound $\left|N_{1}(u)\right| \leqslant \Delta_{1}$ because we work under conditions with $\Delta_{1}$ rather larger than $\Delta_{2}$. Similarly, since $N_{1}^{*}(u) \subset N_{1}(u)$, we need to compensate for the loss of covered vertices by working with the slightly enlarged set $A^{*}(u)$, rather than $A(u)$. The following claims use the condition $\Delta_{1}^{\wedge}<t$ (which is assumed by both theorems).

Claim 3.4. $\left|N_{1}^{*}(u)\right| \leqslant(t-1) \cdot \Delta_{2}$.

Proof. Suppose $\left|N_{1}(u) \cap N_{1}\left(N_{2}(u)\right)\right| \geqslant(t-1) \cdot \Delta_{2}+1$; then there is at least one $x \in N_{2}(u)$ such that

$$
\left|N_{1}(u) \cap N_{1}(x)\right| \geqslant \frac{1}{\left|N_{2}(u)\right|} \cdot\left((t-1) \cdot \Delta_{2}+1\right)>t-1,
$$

which contradicts $\Delta_{1}^{\wedge}<t$.

The following claim (in combination with Corrádi's lemma) is useful for an upper bound on $|B(u)|$ that is only linear in $\Delta_{2}$, provided that $\left|A^{*}(u)\right|$ is at least quadratic in $\Delta_{2}$. See case (i) in the proof of Theorem 1.1.

Claim 3.5. For any $b \in B(u),\left|N_{1}(b) \cap A^{*}(u)\right| \geqslant\left|A^{*}(u)\right| / \Delta_{2}-t\left(\Delta_{2}+1\right)$.

Proof. For all $b \in N_{1}\left(N_{2}(u)\right)$ it holds that

$$
\left|N_{1}(b) \cap N_{1}\left(N_{2}(u)\right)\right| \leqslant(t-1) \cdot\left|N_{2}(u)\right| \leqslant(t-1) \cdot \Delta_{2} .
$$

Indeed, otherwise there would exist a blue copy of $K_{2, t}$ in the graph induced by $N_{1}\left(N_{2}(u)\right) \cup$ $N_{2}(u)$. Similarly, $\left|N_{1}(b) \cap N_{1}(u)\right| \leqslant t$ and $\left|N_{1}(b) \cap N_{2}(u)\right| \leqslant \Delta_{2}$. So for every $b \in N_{1}\left(N_{2}(u)\right)$, at most $t \cdot\left(\Delta_{2}+1\right)$ blue neighbours of $b$ are in $[n] \backslash A(u)$. So in particular, for every $b \in B(u)$, at most $t \cdot\left(\Delta_{2}+1\right)$ blue neighbours of $b$ are in $[n] \backslash A^{*}(u)$.

Using Claim 3.2 and the fact that each blue neighbour of a fixed $b \in B(u)$ has at most $\Delta_{2}$ red neighbours in $A^{*}(u)$, we see that every $b \in B(u)$ has at least $\left\lceil\left|A^{*}(u)\right| / \Delta_{2}\right\rceil$ blue neighbours, and thus at least $\left|A^{*}(u)\right| / \Delta_{2}-t\left(\Delta_{2}+1\right)$ blue neighbours in $A^{*}(u)$.

\section{Proofs}

\subsection{Proof of Theorem 1.1}

Suppose the theorem is false. Consider a critical counterexample, a pair of non-packable graphs $\left(G_{1}, G_{2}\right)$, with $G_{2}$ edge-minimal, satisfying the constraints of the theorem. We distinguish three cases, for each of which we derive an upper bound on $n$, given by one of the inequalities (4.2), (4.4) and (4.10). At least one of these three inequalities should hold, so together they contradict 


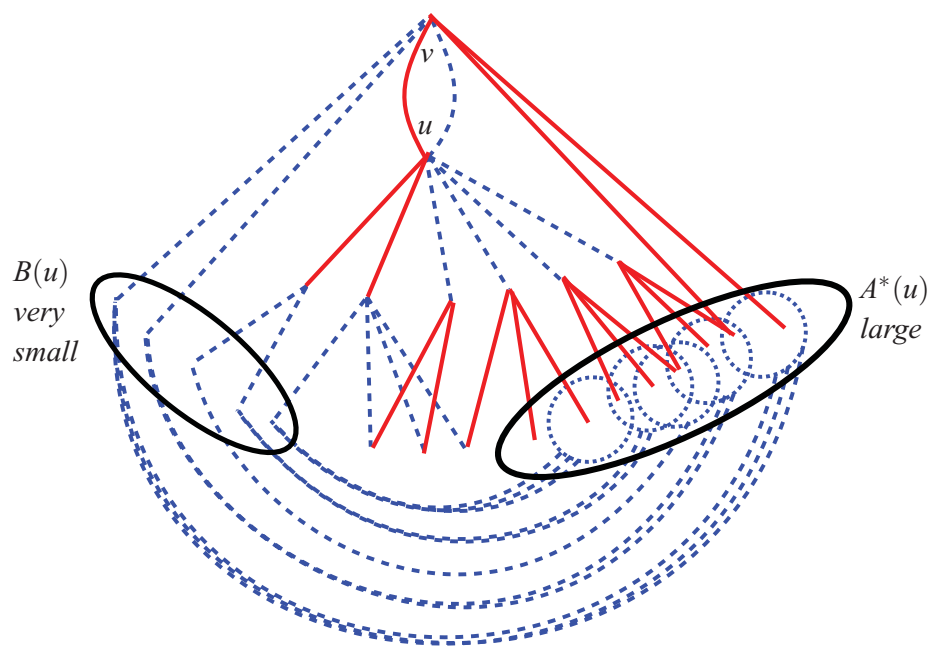

Figure 3. A depiction of case (i) of Theorem 1.1, that $\left|A^{*}(u)\right|=\Omega\left(\Delta_{2}^{2}\right)$ implies $|B(u)|=O\left(\Delta_{2}\right)$.

the condition that

$$
\max ((4.2),(4.4),(4.10))=\max ((1.1),(1.2),(1.3),(1.4))<n,
$$

thus proving the theorem.

(i) There exists a vertex $u \in[n]$ and there are labellings of $G_{1}$ and $G_{2}$ such that $u$ is incident to the unique purple edge and $\left|A^{*}(u)\right| \geqslant \alpha t \cdot \Delta_{2}\left(\Delta_{2}+1\right)$.

(ii) Case (i) does not hold and furthermore $\left|N_{2}(u) \cap N_{2}(v)\right|<(1-\varepsilon) \cdot \Delta_{2}$ for some edge $u v \in$ $E\left(G_{2}\right)$.

(iii) Case (i) does not hold and $\left|N_{2}(u) \cap N_{2}(v)\right| \geqslant(1-\varepsilon) \cdot \Delta_{2}$ for every $u v \in E\left(G_{2}\right)$.

We now proceed with deriving upper bounds on $n$ for each of these three cases.

Bound for case (i). Choose a vertex $u \in[n]$ and labellings of $G_{1}$ and $G_{2}$ such that $u$ is incident to the unique purple edge and $\left|A^{*}(u)\right| \geqslant \alpha t \cdot \Delta_{2}\left(\Delta_{2}+1\right)$. See Figure 3 for a depiction of the argumentation in this case. From now on, we write $k:=\left|A^{*}(u)\right| / \Delta_{2}-t\left(\Delta_{2}+1\right)$. Our first tool is Claim 3.5, which yields that all $b \in B(u)$ satisfy $\left|N_{1}(b) \cap A^{*}(u)\right| \geqslant k$. Note that $k \geqslant 1$, since $\alpha>1$. Our second tool is Corrádi's lemma, or rather Corollary 2.3, which we apply with $X=A^{*}(u)$ and $N=|B(u)|$ and with size $\geqslant k$ subsets $A_{1}, \ldots, A_{N} \subset X$ given by $N_{1}(b) \cap A^{*}(u)$, for all $b \in B(u)$. Note that $\left|A_{i} \cap A_{j}\right| \leqslant t-1$ for all $i \neq j$, or else there would be a blue copy of $K_{2, t}$.

In order to apply Corollary 2.3, we need to check that its condition $k^{2}>(t-1) \cdot\left|A^{*}(u)\right|$ holds. For that, we write $\beta:=\left|A^{*}(u)\right| /\left(t \Delta_{2}\left(\Delta_{2}+1\right)\right)$, so that $k=(\beta-1) t\left(\Delta_{2}+1\right)$. Now

$$
\begin{aligned}
k^{2}-(t-1) \cdot\left|A^{*}(u)\right| & =\left((\beta-1) t\left(\Delta_{2}+1\right)\right)^{2}-\beta t \Delta_{2}\left(\Delta_{2}+1\right)(t-1) \\
& =\left((\beta-1)^{2}-\gamma \cdot \beta\right) \cdot\left(t\left(\Delta_{2}+1\right)\right)^{2},
\end{aligned}
$$

which is positive if and only if $(\beta-1)^{2}-\gamma \beta>0$, which holds true because $\beta \geqslant \alpha>\alpha^{*}$. Thus, by Corollary 2.3, we obtain

$$
|B(u)| \leqslant\left|A^{*}(u)\right| \cdot \frac{k-(t-1)}{k^{2}-(t-1) \cdot\left|A^{*}(u)\right|}=\frac{1-(t-1) / k}{k /\left|A^{*}(u)\right|-(t-1) / k} .
$$


The numerator and denominator of the right-hand side are both positive, so we can bound and rearrange as follows:

$$
\begin{aligned}
|B(u)| & \leqslant\left(\frac{k}{\left|A^{*}(u)\right|}-\frac{t-1}{k}\right)^{-1}=\left(\frac{(\beta-1) t\left(\Delta_{2}+1\right)}{\beta t \Delta_{2}\left(\Delta_{2}+1\right)}-\frac{t-1}{(\beta-1) t\left(\Delta_{2}+1\right)}\right)^{-1} \\
& =\Delta_{2} \cdot\left(\frac{\beta-1}{\beta}-\frac{1}{\beta-1} \cdot \frac{\Delta_{2}}{\Delta_{2}+1} \cdot \frac{t-1}{t}\right)^{-1}=\Delta_{2} \cdot\left(\frac{\beta-1}{\beta}-\frac{\gamma}{\beta-1}\right)^{-1} \\
& \leqslant \Delta_{2} \cdot \frac{\alpha(\alpha-1)}{(\alpha-1)^{2}-\gamma \alpha}
\end{aligned}
$$

where the last step holds because $\beta \geqslant \alpha>\alpha^{*}$ and $\alpha^{*}$ is the larger singular point of

$$
\frac{\beta(\beta-1)}{(\beta-1)^{2}-\gamma \beta},
$$

which is a decreasing function of $\beta$ for all $\beta>\alpha^{*}$.

Evaluating (4.1) and Claim 3.4 in the upper bound of Claim 3.3(ii) yields

$$
\begin{aligned}
n & \leqslant\left|N_{1}^{*}(u)\right|+\left|N_{2}(u)\right|+|B(u)|+\left|N_{2}\left(N_{1}(u)\right)\right| \\
& \leqslant(t-1) \cdot \Delta_{2}+\Delta_{2}+\frac{\alpha(\alpha-1)}{(\alpha-1)^{2}-\alpha} \cdot \Delta_{2}+\Delta_{1} \Delta_{2} \\
& =\left(t+\frac{\alpha(\alpha-1)}{(\alpha-1)^{2}-\alpha}\right) \cdot \Delta_{2}+\Delta_{1} \Delta_{2} .
\end{aligned}
$$

Bound for case (ii). Choose labellings of $G_{1}$ and $G_{2}$ such that there is a unique purple edge $u v$ that satisfies $\left|N_{2}(u) \cap N_{2}(v)\right|<(1-\varepsilon) \cdot \Delta_{2}$. Note that the inequalities $\left|A^{*}(u)\right|<\alpha t \cdot \Delta_{2}\left(\Delta_{2}+1\right)$ and $\left|A^{*}(v)\right|<\alpha t \cdot \Delta_{2}\left(\Delta_{2}+1\right)$ are satisfied as well, as a direct consequence of the assumptions of case (ii).

We proceed with deriving a technical estimate on an intersection of neighbourhood sets. For each $x \in N_{2}(u) \backslash N_{2}(v)$ and $y \in N_{2}(v) \backslash N_{2}(u)$ we have $x \neq y$ and therefore absence of blue copies of $K_{2, t}$ implies the inequality $\left|N_{1}(x) \cap N_{1}(y)\right| \leqslant t-1$. So

$$
\begin{aligned}
\left|N_{1}\left(N_{2}(u) \backslash N_{2}(v)\right) \cap N_{1}\left(N_{2}(v) \backslash N_{2}(u)\right)\right| & \leqslant \sum_{x \in N_{2}(u) \backslash N_{2}(v)} \sum_{y \in N_{2}(v) \backslash N_{2}(u)}\left|N_{1}(x) \cap N_{1}(y)\right| \\
& \leqslant\left|N_{2}(u) \backslash N_{2}(v)\right| \cdot\left|N_{2}(v) \backslash N_{2}(u)\right| \cdot(t-1) \\
& \leqslant\left(\Delta_{2}-\left|N_{2}(u) \cap N_{2}(v)\right|\right)^{2} \cdot(t-1) .
\end{aligned}
$$

Furthermore, since $\left|N_{2}(u) \cap N_{2}(v)\right|<(1-\varepsilon) \cdot \Delta_{2}$,

$$
\begin{aligned}
\left|N_{1}\left(N_{2}(u)\right) \cap N_{1}\left(N_{2}(v)\right)\right| & \leqslant\left|N_{1}\left(N_{2}(u) \cap N_{2}(v)\right)\right|+\left|N_{1}\left(N_{2}(u) \backslash N_{2}(v)\right) \cap N_{1}\left(N_{2}(v) \backslash N_{2}(u)\right)\right| \\
& <\Delta_{1} \cdot\left|N_{2}(u) \cap N_{2}(v)\right|+\left(\Delta_{2}-\left|N_{2}(u) \cap N_{2}(v)\right|\right)^{2} \cdot(t-1) \\
& \leqslant \max _{p \in\left\{0,1,2, \ldots,\left\lfloor(1-\varepsilon) \cdot \Delta_{2}\right\rfloor\right\}}\left(\Delta_{1} \cdot p+\left(\Delta_{2}-p\right)^{2} \cdot(t-1)\right) .
\end{aligned}
$$




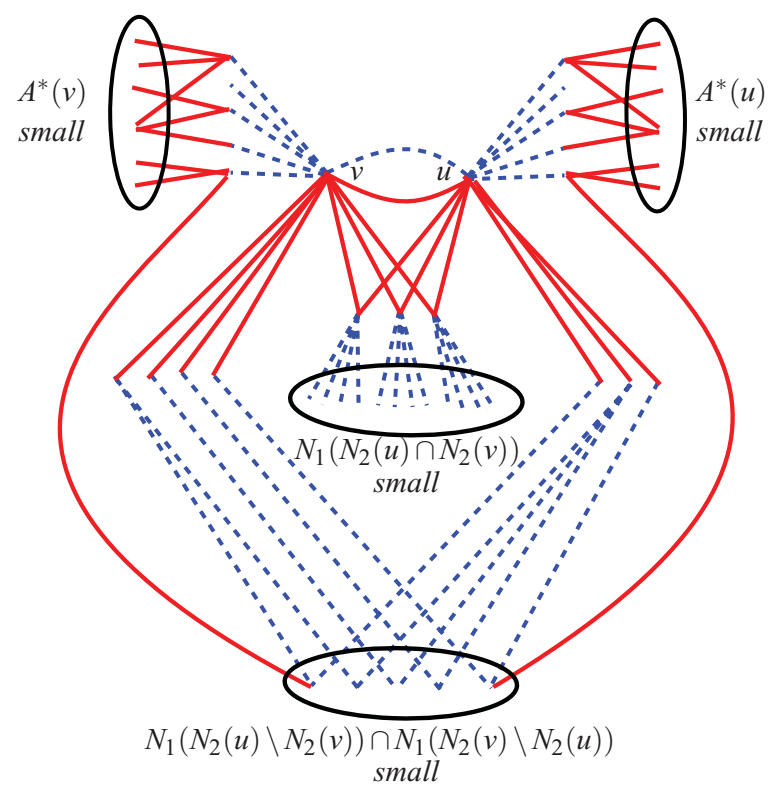

Figure 4. A depiction of case (ii) of Theorem 1.1, that $\left|N_{1}\left(N_{2}(u)\right) \cap N_{1}\left(N_{2}(v)\right)\right|$ is small.

See Figure 4. Finally, we evaluate this in Claim 3.3(iii) to find the following bound on $n$ :

$$
\begin{aligned}
n & \leqslant\left|A^{*}(v)\right|+\left|A^{*}(u)\right|+\left|\left(N_{2}(u) \cup N_{1}\left(N_{2}(u)\right)\right) \cap\left(N_{2}(v) \cup N_{1}\left(N_{2}(v)\right)\right)\right| \\
& \leqslant\left|A^{*}(v)\right|+\left|A^{*}(u)\right|+\left|N_{2}(u)\right|+\left|N_{2}(v)\right|+\left|N_{1}\left(N_{2}(u)\right) \cap N_{1}\left(N_{2}(v)\right)\right| \\
& \leqslant 2 \alpha t \cdot \Delta_{2}\left(\Delta_{2}+1\right)+2 \Delta_{2}+\max _{p \in\left\{0,1,2, \ldots,\left\lfloor(1-\varepsilon) \cdot \Delta_{2}\right\rfloor\right\}}\left(\Delta_{1} \cdot p+\left(\Delta_{2}-p\right)^{2} \cdot(t-1)\right) .
\end{aligned}
$$

In particular, this implies the slightly rougher bound

$$
n \leqslant 2 \alpha t \cdot \Delta_{2}\left(\Delta_{2}+1\right)+2 \Delta_{2}+(1-\varepsilon) \cdot \Delta_{1} \Delta_{2}+\Delta_{2}^{2} \cdot(t-1) .
$$

Bound for case (iii). Choose a pair of labellings of $G_{1}$ and $G_{2}$ that induces a unique purple edge $u v$. The assumptions of this case imply, in particular, that in the red graph the neighbourhoods of each pair of adjacent vertices overlap significantly: $\left|N_{2}(x) \cap N_{2}(y)\right| \geqslant(1-\varepsilon) \cdot \Delta_{2}$ for each $x y \in E\left(G_{2}\right)$.

We will derive two consequences, namely the implication

$$
\left(\left|A^{*}(u)\right| \geqslant 1+\Delta_{2}+\frac{\varepsilon \cdot \Delta_{2}}{1-2 \varepsilon}\right) \Longrightarrow\left(|B(u)| \leqslant(t-1) \cdot \Delta_{2}^{2}\right)
$$

and the inequality

$$
\left|N_{2}\left(N_{1}(u)\right)\right| \leqslant \frac{1+\varepsilon}{2} \Delta_{1} \Delta_{2}+\frac{1-\varepsilon}{2}(t-1) \cdot \Delta_{2}^{2}+\frac{3}{2} \Delta_{2} .
$$

We start by proving the statement (4.5), the first consequence. See Figure 5. Suppose $a \in$ $A^{*}(u) \backslash N_{2}(u)$ has a red neighbour $x \in N_{2}(u)$. Then $u x$ and $a x$ are edges of $G_{2}$, so $\left|N_{2}(a) \cap N_{2}(x)\right| \geqslant$ $(1-\varepsilon) \Delta_{2}$ and $\left|N_{2}(u) \cap N_{2}(x)\right| \geqslant(1-\varepsilon) \Delta_{2}$. Combining this with the obvious fact that $\left|N_{2}(x)\right| \leqslant \Delta_{2}$ 


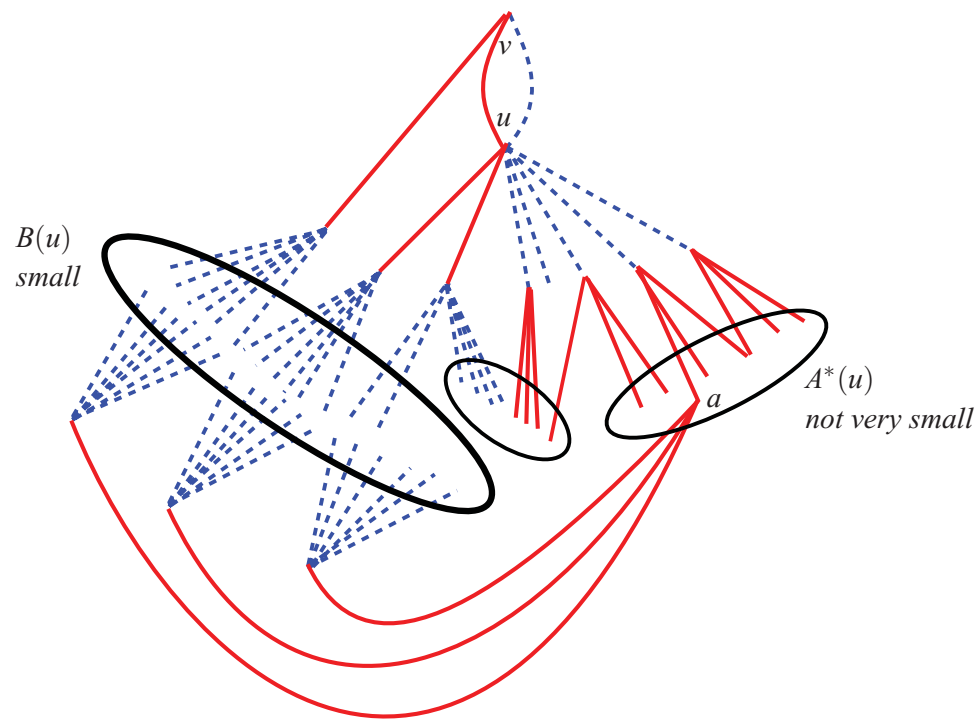

Figure 5. A depiction of (4.5) in case (iii) of Theorem 1.1.

yields that

$$
\left|N_{2}(a) \cap N_{2}(u)\right| \geqslant(1-2 \varepsilon) \cdot \Delta_{2} .
$$

Let us define

$$
A^{* *}(u):=\left\{a \in A^{*}(u) \mid a \text { has a red neighbour in } N_{2}(u)\right\} .
$$

It follows from (4.7) that

$$
\sum_{a \in A^{* *}(u)}\left|N_{2}(a) \cap N_{2}(u)\right| \geqslant\left|A^{* *}(u)\right| \cdot(1-2 \varepsilon) \cdot \Delta_{2},
$$

so

$$
\begin{aligned}
\sum_{x \in N_{2}(u)}\left|N_{2}(x)\right| & \geqslant \sum_{x \in N_{2}(u)}\left|N_{2}(x) \cap N_{2}(u)\right|+\sum_{a \in A^{* *}(u)}\left|N_{2}(a) \cap N_{2}(u)\right| \\
& \geqslant(1-\varepsilon) \Delta_{2} \cdot\left|N_{2}(u)\right|+\left|A^{* *}(u)\right| \cdot(1-2 \varepsilon) \cdot \Delta_{2},
\end{aligned}
$$

and (crucially) since $\sum_{x \in N_{2}(u)}\left|N_{2}(x)\right| \leqslant \Delta_{2} \cdot\left|N_{2}(u)\right|$, it follows that

$$
\left|A^{* *}(u)\right| \leqslant \frac{\left|N_{2}(u)\right| \cdot \Delta_{2}-(1-\varepsilon) \cdot \Delta_{2}\left|N_{2}(u)\right|}{(1-2 \varepsilon) \cdot \Delta_{2}}=\frac{\varepsilon \cdot\left|N_{2}(u)\right|}{1-2 \varepsilon} .
$$

Next, suppose we would have that $\left|A^{*}(u)\right| \geqslant 1+\left|N_{2}(u)\right|+\left|A^{* *}(u)\right|$. Then there exists a vertex $a \in A^{*}(u) \backslash A^{* *}(u)$. By the definition of $A^{* *}(u)$, this vertex satisfies $N_{2}(a) \cap N_{2}(u)=\emptyset$. Furthermore, since $a \in A^{*}(u)$, we have that for all $b \in B(u)$ there is a red-blue link from $a$ to $b$. In other words, $B(u)=N_{1}\left(N_{2}(a)\right) \cap B(u)$. This implies that

$$
|B(u)|=\left|N_{1}\left(N_{2}(a)\right) \cap B(u)\right| \leqslant\left|N_{1}\left(N_{2}(a)\right) \cap N_{1}\left(N_{2}(u)\right)\right| \leqslant(t-1) \cdot \Delta_{2}^{2},
$$


where the last inequality is a consequence of the facts that $N_{2}(a) \cap N_{2}(u)=\emptyset$ and $G_{1}$ does not contain a copy of $K_{2, t}$. In summary, we have shown the implication

$$
\left|A^{*}(u)\right| \geqslant 1+\left|N_{2}(u)\right|+\left|A^{* *}(u)\right| \Longrightarrow|B(u)| \leqslant(t-1) \cdot \Delta_{2}^{2} .
$$

Combining (4.8) and (4.9) yields our first desired main consequence (4.5).

We now prove inequality (4.6), the second consequence. See Figure 6. First, the absence of blue copies of $K_{2, t}$ implies that for every $x \in N_{2}(u)$ we have $\left|N_{1}(x) \cap N_{1}(u)\right| \leqslant t-1$. Therefore

$$
\left|N_{1}(u) \cap N_{1}\left(N_{2}(u)\right)\right| \leqslant\left|N_{2}(u)\right| \cdot \max _{x \in N_{2}(u)}\left(\left|N_{1}(x) \cap N_{1}(u)\right|\right) \leqslant \Delta_{2} \cdot(t-1) .
$$

In other words, there is a red-blue link from $u$ to $y$ for at most $\Delta_{2} \cdot(t-1)$ vertices $y \in N_{1}(u)$. Recalling that there is a link from $u$ to every vertex (possibly with the exception of $v$ ), it follows that there are at least $h:=\left|N_{1}(u)\right|-(t-1) \Delta_{2}-1$ vertices $y \in N_{1}(u)$ for which there is a blue-red link (and no red-blue link) from $u$ to $y$. In other words, $m:=\left|N_{1}(u) \cap N_{2}\left(N_{1}(u)\right)\right| \geqslant h$. It follows from the definition of blue-red link that any $y_{1} \in N_{1}(u) \cap N_{2}\left(N_{1}(u)\right)$ is connected to at least one other vertex $y_{2} \in N_{1}(u) \cap N_{2}\left(N_{1}(u)\right)$ by a red edge.

This means that $N_{1}(u) \cap N_{2}\left(N_{1}(u)\right)$ can be covered by a collection of vertex-disjoint red stars $S_{1}, S_{2}, \ldots$ that each have at least two vertices (unless $m \in\{0,1\}$, in which case inequality (4.6) is clearly satisfied). Let $S$ be one such star, with central vertex $y^{*}$ and leaves $y_{1}, y_{2} \ldots, y_{|S|-1}$. Each of its edges has a large common red neighbourhood: for all $j \in\{1,2, \ldots,|S|-1\}$ it holds that $\left|N_{2}\left(y^{*}\right) \cap N_{2}\left(y_{j}\right)\right| \geqslant(1-\varepsilon) \cdot \Delta_{2}$. Therefore

$$
\left|\bigcup_{y \in S} N_{2}(y)\right| \leqslant\left|N_{2}\left(y^{*}\right)\right|+\sum_{y \in S \backslash\left\{y^{*}\right\}}\left|N_{2}(y) \backslash N_{2}\left(y^{*}\right)\right| \leqslant(1+\varepsilon \cdot(|S|-1)) \cdot \Delta_{2},
$$

which is at most $(1+\varepsilon) / 2 \cdot|S| \cdot \Delta_{2}$. So

$$
\begin{aligned}
\left|N_{2}\left(N_{1}(u) \cap N_{2}\left(N_{1}(u)\right)\right)\right| & =\left|\bigcup_{i} \bigcup_{y \in S_{i}} N_{2}(y)\right| \leqslant \sum_{i}\left|\bigcup_{y \in S_{i}} N_{2}(y)\right| \\
& \leqslant \sum_{i} \frac{1+\varepsilon}{2} \cdot\left|S_{i}\right| \cdot \Delta_{2}=\frac{m}{2} \cdot(1+\varepsilon) \cdot \Delta_{2} .
\end{aligned}
$$

Last, note that

$$
\left|N_{1}(u) \cap\left(N_{1}\left(N_{2}(u)\right) \backslash N_{2}\left(N_{1}(u)\right)\right)\right|=\left|N_{1}(u)\right|-m-\mathbb{1}_{\{\nexists \text { link from } u \text { to } v\}} \leqslant\left|N_{1}(u)\right|-m .
$$

We are now ready to derive (4.6):

$$
\begin{aligned}
\left|N_{2}\left(N_{1}(u)\right)\right| & \leqslant\left|N_{2}\left(N_{1}(u) \cap N_{2}\left(N_{1}(u)\right)\right)\right|+\left|N_{2}\left(N_{1}(u) \cap\left(N_{1}\left(N_{2}(u)\right) \backslash N_{2}\left(N_{1}(u)\right)\right)\right)\right|+\left|N_{2}(v)\right| \\
& \leqslant \frac{m}{2} \cdot(1+\varepsilon) \cdot \Delta_{2}+\left(\left|N_{1}(u)\right|-m\right) \cdot \Delta_{2}+\Delta_{2}=: g(m) .
\end{aligned}
$$




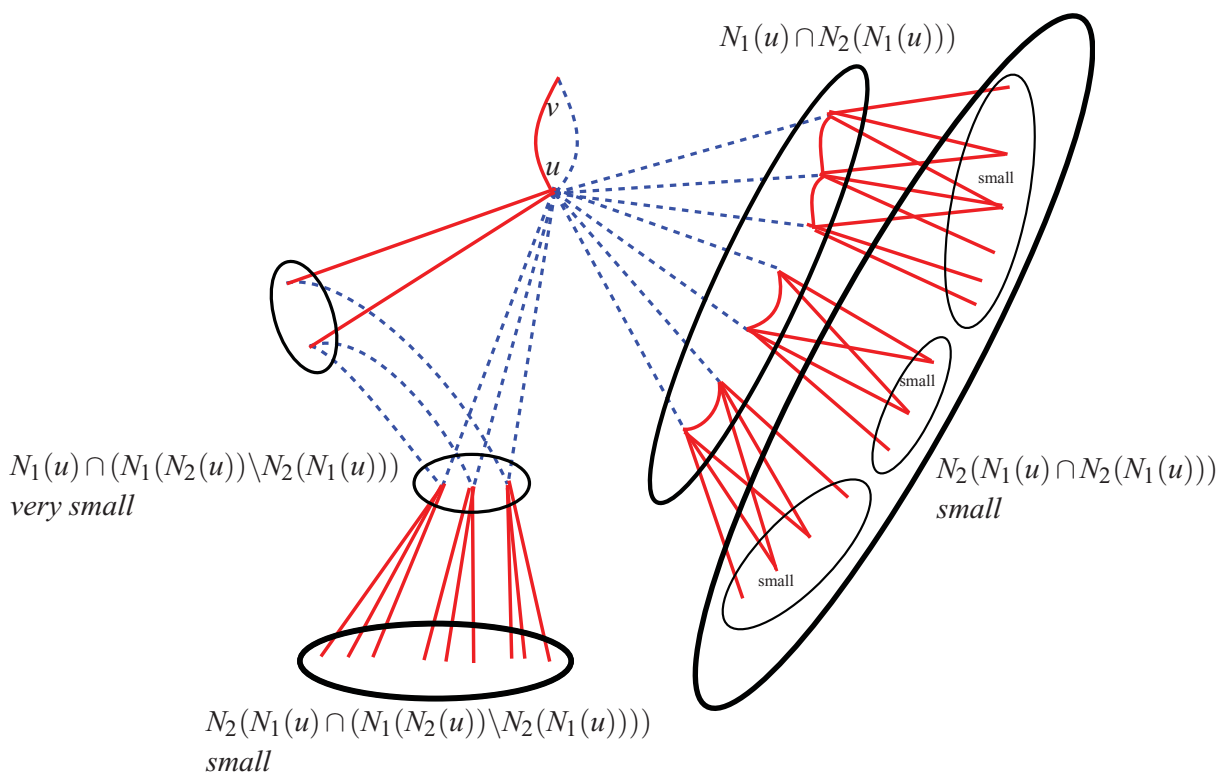

Figure 6. A depiction of (4.6) in case (iii) of Theorem 1.1.

Since $\Delta_{2} \geqslant 0$ and $\varepsilon<1 / 2$, the function $g(x)$ is non-increasing on the whole of $\mathbb{R}$. Since $h \leqslant m$, it follows that $g(m) \leqslant g(h)$. So

$$
\begin{aligned}
\left|N_{2}\left(N_{1}(u)\right)\right| & \leqslant g\left(\left|N_{1}(u)\right|-(t-1) \Delta_{2}-1\right) \\
& =\frac{1+\varepsilon}{2} \cdot\left(\left|N_{1}(u)\right|-(t-1) \cdot \Delta_{2}-1\right) \cdot \Delta_{2}+(t-1) \cdot \Delta_{2}^{2}+2 \Delta_{2} \\
& \leqslant \frac{1+\varepsilon}{2} \cdot \Delta_{1} \Delta_{2}+\frac{1-\varepsilon}{2} \cdot(t-1) \cdot \Delta_{2}^{2}+\frac{3-\varepsilon}{2} \cdot \Delta_{2},
\end{aligned}
$$

as desired.

Finally, we evaluate (4.5) and (4.6) in the bounds on $n$ given by Claim 3.3, parts (i) and (ii), to obtain

$$
\begin{aligned}
n & \leqslant \min \left(\left|N_{1}\left(N_{2}(u)\right)\right|+\left|A^{*}(u)\right|+\left|N_{2}(u)\right|,\left|N_{2}\left(N_{1}(u)\right)\right|+\left|N_{2}(u)\right|+\left|N_{1}^{*}(u)\right|+|B(u)|\right) \\
& \leqslant \min \left(\Delta_{1} \Delta_{2}+\Delta_{2}+\left|A^{*}(u)\right|, \frac{1+\varepsilon}{2} \Delta_{1} \Delta_{2}+\frac{1-\varepsilon}{2}(t-1) \Delta_{2}^{2}+\left(t+\frac{3-\varepsilon}{2}\right) \cdot \Delta_{2}+|B(u)|\right) \\
& =\Delta_{1} \Delta_{2}+\Delta_{2}+\min \left(\left|A^{*}(u)\right|,|B(u)|+\left(t+\frac{1-\varepsilon}{2}\right) \cdot \Delta_{2}-\frac{1-\varepsilon}{2}\left(\Delta_{1} \Delta_{2}-(t-1) \Delta_{2}^{2}\right)\right) \\
& \leqslant \Delta_{1} \Delta_{2}+\Delta_{2}+\max \left(1+\Delta_{2}+\frac{\varepsilon \Delta_{2}}{1-2 \varepsilon}, \frac{3-\varepsilon}{2}(t-1) \cdot \Delta_{2}^{2}-\frac{1-\varepsilon}{2} \Delta_{1} \Delta_{2}+\left(t+\frac{1-\varepsilon}{2}\right) \cdot \Delta_{2}\right)
\end{aligned}
$$

where we employed Claim 3.3 in the first line, Claim 3.4 and inequality (4.6) in the second line and implication (4.5) in the last line. 


\subsection{Proof of Theorem 1.2}

Suppose the theorem is false. Consider a critical counterexample, a pair of non-packable graphs $\left(G_{1}, G_{2}\right)$ satisfying the constraints of the theorem, such that there is a near-packing with a unique purple edge $u v$. We distinguish two cases, (i) and (ii). From the first we derive the inequality (4.11) and from the second we obtain the inequality (4.12). Together they contradict the condition that $\max ((1.5),(1.6))<n$, thus proving the theorem.

(i) $\left|A^{*}(u)\right| \geqslant \alpha t \cdot \Delta_{2}\left(\Delta_{2}+1\right)$ or $\left|A^{*}(v)\right| \geqslant \alpha t \cdot \Delta_{2}\left(\Delta_{2}+1\right)$.

Without loss of generality, we assume $\left|A^{*}(u)\right| \geqslant \alpha t \cdot \Delta_{2}\left(\Delta_{2}+1\right)$. From here on the proof is the same as for case (i) in the proof of Theorem 1.1, leading to the same bound,

$$
n \leqslant\left(t+\frac{\alpha(\alpha-1)}{(\alpha-1)^{2}-\alpha}\right) \cdot \Delta_{2}+\Delta_{1} \Delta_{2} .
$$

(ii) Case (i) does not hold.

From here on we proceed almost exactly as for case (ii) in the proof of Theorem 1.1, the difference being that instead of the upper bound $\left|N_{2}(u) \cap N_{2}(v)\right|<(1-\varepsilon) \cdot \Delta_{2}$ we use $\mid N_{2}(u) \cap$ $N_{2}(v) \mid<s$, which holds due to the additional condition $\Delta_{2}^{\Delta}<s$. (Compare with (4.4).) It follows that

$$
n \leqslant 2 \alpha t \cdot \Delta_{2}\left(\Delta_{2}+1\right)+2 \Delta_{2}+\Delta_{1} \cdot(s-1)+\Delta_{2}^{2} \cdot(t-1) .
$$

\subsection{Concluding remarks}

We wish to make the following remarks about Theorems 1.1 and 1.2.

- In Theorem 1.1, the bottleneck is the quantity (1.2), which corresponds to the bound (4.4) of case (ii). So improving in this case would improve the overall bound on $n$, albeit not by much.

- The condition in Theorem 1.2 that $\Delta_{2}^{\Delta}<s$ is equivalent to ' $\left|N_{2}(x) \cap N_{2}(y)\right|<s$ for all $x y \in$ $E\left(G_{2}\right)$ '. With a little adaptation, we can replace this with the weaker but perhaps obscure condition that $G_{2}$ has no subgraph $G_{2}^{!}$such that $\left|N_{2}(x) \cap N_{2}(y)\right| \geqslant s$ for all $x y \in E\left(G_{2}^{!}\right)$. Indeed, this property is invariant under edge removal, and so holds for an edge-minimal critical counterexample, which therefore has an edge $u v$ with $|N(u) \cap N(v)|<s$, for which we can choose labellings such that $u v$ is the unique purple edge. From here on, one again proceeds exactly as in case (ii) of the proof of Theorem 1.1.

- Theorem 1.2 yields a better bound than Theorem 1.1 only if $\Delta_{1}$ is much larger than $\Delta_{2}$ and $s$, $t$ are both small.

- By taking $G_{2}$ to be a collection of (nearly) equal-sized cliques, Corollary 1.4 implies that if $G$ is a $K_{2, t}$-free graph of maximum degree $\Delta$ with $\Delta \geqslant \sqrt{17 t} \cdot \sqrt{n}$, then the equitable chromatic number of $G$ is at most $\Delta$. Note that this result cannot be obtained by the result of Hajnal and Szemerédi on equitable colourings [12].

The BEC conjecture notwithstanding, naturally one might wonder whether Theorem 1.1, or rather Corollary 1.5, could be improved according to a weaker form of the BEC condition, as was the case for $d$-degenerate $G_{1}$ [5]. In other words, it would be interesting to improve upon the $\Omega\left(\Delta_{1} \Delta_{2}\right)$ terms appearing in each of (1.1)-(1.4). We leave this to further study, but point out the 
following constructions where $G_{1}$ has low maximum codegree, which mark boundaries for this problem.

- When $n$ is even, there are non-packable pairs $\left(G_{1}, G_{2}\right)$ of graphs where $G_{1}$ is a perfect matching (so $\Delta_{1}^{\wedge}=0$ ) and $2 \Delta_{1} \Delta_{2}=n$ : see [14].

- Bollobás, Kostochka and Nakprasit [4] exhibited a family of non-packable pairs $\left(G_{1}, G_{2}\right)$ of graphs where $G_{1}$ is a forest (so $\Delta_{1}^{\wedge}=1$ ) and $\Delta_{1} \ln \Delta_{2} \geqslant c n$ for some $c>0$.

- If $\Delta^{\wedge}(G)=1$, then the chromatic number of $G$ satisfies $\chi(G)=O(\Delta(G) / \ln \Delta(G))$ as $\Delta(G) \rightarrow$ $\infty$, and there are standard examples having arbitrarily large girth that show this bound to be sharp up to a constant factor: see [16, Ex. 12.7]. Since the equitable chromatic number is at least the chromatic number, these examples moreover yield non-packable pairs $\left(G_{1}, G_{2}\right)$ of graphs having $\left(\Delta_{1} / \ln \Delta_{1}\right)\left(\Delta_{2}+1\right) \geqslant c n$ for some $c>0$ and $\Delta_{1}^{\wedge}=1$.

Since the examples can also have the maximum adjacent codegree $\Delta_{1}^{\Delta}$ being zero, this last remark hints at another natural line to pursue, which could significantly extend both the result of Csaba [9] and a result of Johansson [13]. If $\Delta_{1}$ is large enough and $G_{1}$ is triangle-free, is some condition of the form $\left(\Delta_{1} / \ln \Delta_{1}\right)\left(\Delta_{2}+1\right) \leqslant c n$ for some constant $c>0$ sufficient for $G_{1}$ and $G_{2}$ to pack?

\section{Acknowledgement}

We are grateful to the anonymous referee for their careful reading and helpful comments.

\section{References}

[1] Aigner, M. and Brandt, S. (1993) Embedding arbitrary graphs of maximum degree two. J. London Math. Soc. (2) 48 39-51.

[2] Bollobás, B. and Eldridge, S. E. (1978) Packings of graphs and applications to computational complexity. J. Combin. Theory Ser. B 25 105-124.

[3] Bollobás, B., Janson, S. and Scott, A. (2017) Packing random graphs and hypergraphs. Random Struct. Alg. 513-13.

[4] Bollobás, B., Kostochka, A. and Nakprasit, K. (2005) On two conjectures on packing of graphs. Combin. Probab. Comput. 14 723-736.

[5] Bollobás, B., Kostochka, A. and Nakprasit, K. (2008) Packing $d$-degenerate graphs. J. Combin. Theory Ser. B 98 85-94.

[6] Catlin, P. A. (1974) Subgraphs of graphs I. Discrete Math. 10 225-233.

[7] Catlin, P. A. (1976) Embedding subgraphs and coloring graphs under extremal degree conditions. PhD thesis, The Ohio State University. ProQuest LLC, Ann Arbor, MI.

[8] Corrádi, K. (1969) Problem at Schweitzer competition. Mat. Lapok 20 159-162.

[9] Csaba, B. (2007) On the Bollobás-Eldridge conjecture for bipartite graphs. Combin. Probab. Comput. 16 661-691.

[10] Csaba, B., Shokoufandeh, A. and Szemerédi, E. (2003) Proof of a conjecture of Bollobás and Eldridge for graphs of maximum degree three. Combinatorica 23 35-72.

[11] Eaton, N. (2000) A near packing of two graphs. J. Combin. Theory Ser. B 80 98-103.

[12] Hajnal, A. and Szemerédi, E. (1970) Proof of a conjecture of P. Erdős. In Combinatorial Theory and its Applications II: (Proc. Colloq., Balatonfüred, 1969), North-Holland, pp. 601-623.

[13] Johansson, A. (1996) Asymptotic choice number for triangle-free graphs. Technical report 91-5, DIMACS. 
[14] Kaul, H. and Kostochka, A. (2007) Extremal graphs for a graph packing theorem of Sauer and Spencer. Combin. Probab. Comput. 16 409-416.

[15] Kaul, H., Kostochka, A. and Yu, G. (2008) On a graph packing conjecture by Bollobás, Eldridge and Catlin. Combinatorica 28 469-485.

[16] Molloy, M. and Reed, B. (2002) Graph Colouring and the Probabilistic Method, Vol. 23 of Algorithms and Combinatorics, Springer.

[17] Sauer, N. and Spencer, J. (1978) Edge disjoint placement of graphs. J. Combin. Theory Ser. B 25 295-302. 